NBI-HE-96-xx

June 1996

\title{
New universal spectral correlators
}

\author{
J. Ambjørn and G. Akemann円 \\ The Niels Bohr Institute \\ Blegdamsvej 17, DK-2100 Copenhagen Ø, Denmark
}

\begin{abstract}
We study the universal properties of distributions of eigenvalues of random matrices in the large $N$ limit. The distributions fall in universality classes characterized entirely by the support of the spectral density.
\end{abstract}

\footnotetext{
${ }^{1}$ Address after June 96: Inst.f.Theor.Physik, Appelstr.2, D-30167 Hannover, Germany
} 


\section{The theorems}

Random matrices have found a wide range of applications in solid state physics, nuclear physics and high energy physics. An important observable in most of these applications is the correlator between eigenvalues of the random matrices. In addition to being random, the matrices are often large, and it is natural to take the so called large $N$ limit in many of the applications. The general set up is as follows: we consider an ensemble of random matrices

$$
P(\phi)=\frac{1}{Z} \mathrm{e}^{-N \operatorname{Tr} V(\phi)}, \quad Z=\int d \phi \mathrm{e}^{-N \operatorname{Tr} V(\phi)}, \quad V(\phi)=\sum_{k} \frac{g_{k}}{k} \phi^{k}
$$

and in this ensemble we can ask for expectation values of a certain observables :

$$
\langle f(\phi)\rangle=\int d \phi P(\phi) f(\phi) .
$$

In the following we will assume that the random matrices $\phi$ are $N \times N$ Hermitian matrices, but similar results can be proven for ensembles of complex matrices matrices.

The one- and two-point "resolvents" are defined by

$$
\begin{aligned}
G(z) & =\frac{1}{N}\left\langle\operatorname{Tr} \frac{1}{z-\phi}\right\rangle \\
G\left(z_{1}, z_{2}\right) & =\left\langle\operatorname{Tr} \frac{1}{z_{1}-\phi} \operatorname{Tr} \frac{1}{z_{2}-\phi}\right\rangle-\left\langle\operatorname{Tr} \frac{1}{z_{1}-\phi}\right\rangle\left\langle\operatorname{Tr} \frac{1}{z_{2}-\phi}\right\rangle,
\end{aligned}
$$

They are related to the spectral density and the correlator of densities

$$
\rho(\lambda) \equiv\langle S(\lambda)\rangle, \quad S(\lambda) \equiv \frac{1}{N} \sum_{i=1}^{N} \delta\left(\lambda-\lambda_{i}\right)
$$

and

in the following way

$$
\rho\left(\lambda, \lambda^{\prime}\right) \equiv\left\langle S(\lambda) S\left(\lambda^{\prime}\right)\right\rangle-\langle S(\lambda)\rangle\left\langle S\left(\lambda^{\prime}\right)\right\rangle
$$

$$
\rho(\lambda)=\frac{-1}{2 \pi i}(G(\lambda+i \varepsilon)-G(\lambda-i \varepsilon))
$$

and

$$
\rho\left(\lambda, \lambda^{\prime}\right)=\frac{1}{N^{2}}\left(\frac{-1}{2 \pi i}\right)^{2}(G(+,+)+G(-,-)-G(+,-)-G(-,+)),
$$

where $G( \pm, \pm) \equiv G\left(\lambda \pm i \varepsilon, \lambda^{\prime} \pm i \varepsilon^{\prime}\right)$.

The following theorem was proven in [1] (and partly rediscovered by the authors of [2], after whom it is sometimes called Brezin-Zee universality):

Theorem 1: Assume that the spectral density $\rho(\lambda)$ has support in a single interval in the large $N$ limit. Then the two-point correlator (and thus $\rho\left(\lambda, \lambda^{\prime}\right)$ ) is universal, i.e. independent of $V(\phi)$, and given by (in the case of a symmetric potential, such that the support is $[-a, a]$ )

$$
\begin{aligned}
G\left(z_{1}, z_{2}\right)= & \frac{1}{4\left(z_{1}-z_{2}\right)^{2}}\left(-2+\frac{\left(z_{1}^{2}-a^{2}\right)+\left(z_{2}^{2}-a^{2}\right)}{\sqrt{\left(z_{1}^{2}-a^{2}\right)\left(z_{2}^{2}-a^{2}\right)}}\right) \\
& -\frac{1}{4} \frac{1}{\sqrt{\left(z_{1}^{2}-a^{2}\right)\left(z_{2}^{2}-a^{2}\right)}} .
\end{aligned}
$$


For the general formula for an arbitrary potential, as well as for complex matrices, rather than Hermitian matrices, we refer to [1]. In fact much more was proven in [1]. Any multi-point resolvent

$$
G\left(z_{1}, \ldots, z_{n}\right) \equiv N^{2 n-2}\left\langle\frac{1}{N} \operatorname{Tr} \frac{1}{z_{1}-\phi} \cdots \frac{1}{N} \operatorname{Tr} \frac{1}{z_{n}-\phi}\right\rangle_{c o n n},
$$

where conn refers to the connected part of the multi-point resolvent, is also universal. Closed and very simple expressions were given for the multi-point resolvents in the case where matrix ensemble consisted of complex matrices. Note that the generalization of (8) is

$$
\left\langle S\left(\lambda_{1}\right) \cdots S\left(\lambda_{n}\right)\right\rangle_{\text {conn }}=\frac{1}{N^{2 n-2}}\left(\frac{-1}{2 \pi i}\right)^{n} \sum_{\sigma_{k}= \pm 1}(-1)^{\Sigma_{k} \sigma_{k}} G\left(z_{1}+\sigma_{1} i \varepsilon, \ldots, z_{n}+\sigma_{n} i \varepsilon\right) .
$$

In addition it is possible to develop a systematic $1 / N^{2}$ expansion:

$$
\begin{aligned}
G\left(z_{1}, \ldots, z_{n}\right) & =\sum_{h=0}^{\infty} \frac{1}{N^{2 h}} G_{h}\left(z_{1}, \ldots, z_{n}\right), \\
F & =\sum_{h=0}^{\infty} \frac{1}{N^{2 h}} F_{h} .
\end{aligned}
$$

Again the two-point resolvents and in this case even the corrections to the spectral density $\rho(\lambda)$ as well as the corrections to the free energy $N^{2} F=\log Z$ are all universal [3]. These considerations have later been extended to supermatrices 沺.

In the above discussion universality means that the resolvents can be presented in a form independent of the potential. Of course the function (9) depends on the potential through the endpoints of the eigenvalue distribution $\rho(\lambda)$. The same remark is true for the multipoint resolvents and for higher order expansions in $1 / N^{2}$. However, for the $n$-point resolvent calculated to non-trivial order $h$ in the $1 / N^{2}$ expansion only $2(3 h-2+n)$ additional parameters $M_{1,2}^{(l)}$ are involved for an arbitrary potential (see def. (27)). Yet, some of these might vanish, as will be the case for a Gaussian potential where all additional parameters vanish but one. The somewhat misleading statement found in [2] that all multi-point density correlators vanish to order $1 / N^{n}$ is a trivial fact which follows from the factorization property of the large $N$ expansion. The first non-trivial term is of order $1 / N^{2 n-2}$ for the $n$-point density correlator (11) and it is explicitly non-Gaussian in nature for $n>2$ because of the additional parameters. These observations can be summarized as follows 3:

Theorem 2: If the spectral density in the large $N$ limit has support $[-a, a]$ all higher $1 / N^{2}$ corrections to the free energy $F$, to the $n$-point resolvents, to the spectral density and any multiple eigenvalue correlators are universal. The correction to the $n$-points resolvents are of the form

$$
G_{h}\left(z_{1}, \ldots, z_{n}\right)=\frac{R_{h}\left(\left\{z_{i}^{2}-a^{2}\right\},\left\{M_{a}^{(l)}\right\}, a\right)}{\sqrt{\prod_{i=1}^{n}\left(z_{i}^{2}-a^{2}\right)}},
$$

where the $R_{h}$ are simple rational functions of the arguments, which can be determined iteratively in the $1 / N^{2}$ expansion. 
We refer to [3] for details, generalizations to the situation where the support is not symmetric and to [5] for complex matrix models.

All these considerations have been based on the assumption that $\rho(\lambda)$ has support in one interval on the real axis. More general situations can occur for complicated potentials, and in fact it is natural for applications in solid state physics to have such a situation since the eigenvalues of $\phi$ are viewed as (part of) the eigenvalues of a Hamiltonian which can have a band structure. In this paper we prove

Theorem 3: Assume that the support of the spectral density $\rho(\lambda)$ in the large $N$ limit consists of $s$ intervals $\left[x_{2 j}, x_{2 j-1}\right], j=1, \ldots, s$. The large $N$ limit of the two-point resolvents $G_{0}\left(z_{1}, z_{2}\right)$ (and therefore the correlators of eigenvalues) fall in universality classes characterized by $s$ and they depend only on the potential $V$ through the endpoints $x_{i}$. For each $s>1$ it is possible to generalize the statements in Theorem 1 and 2 concerning universality.

Let us here present the explicit solution, analogous to (9), in the case where the support of $\rho(\lambda)$ consists of two intervals $\left[x_{4}, x_{3}\right]$ and $\left[x_{2}, x_{1}\right], x_{4}<x_{3}<x_{2}<x_{1}$, and where we for simplicity assume that $V(\phi)$ is an even function of $\phi$ such that $x_{4}=-x_{1}$ and $x_{3}=-x_{2}$. If we denote $x_{1}, x_{2}$ by $a, b$ we have

$$
\begin{aligned}
G_{0}\left(z_{1}, z_{2}\right)= & \frac{1}{4\left(z_{1}-z_{2}\right)^{2}}\left(-2+\frac{\left(z_{1}^{2}-a^{2}\right)\left(z_{2}^{2}-b^{2}\right)+\left(z_{1}^{2}-b^{2}\right)\left(z_{2}^{2}-a^{2}\right)}{\sqrt{\left(z_{1}^{2}-a^{2}\right)\left(z_{1}^{2}-b^{2}\right)\left(z_{2}^{2}-a^{2}\right)\left(z_{2}^{2}-b^{2}\right)}}\right) \\
& +\frac{(a+b)^{2}}{4} \frac{E(k)}{K(k)} \frac{1}{\sqrt{\left(z_{1}^{2}-a^{2}\right)\left(z_{1}^{2}-b^{2}\right)\left(z_{2}^{2}-a^{2}\right)\left(z_{2}^{2}-b^{2}\right)}},
\end{aligned}
$$

where $k=2 \sqrt{a b} /(a+b)$, and where $E(k)$ and $K(k)$ denote the complete elliptic integrals of first and second kind.

In the next section we will briefly outline how to prove theorem 3.

\section{Proofs}

The basic tool for proving the above statements is the loop equation in the following form $($ [3]):

$$
\hat{\mathcal{K}} G(z)=G^{2}(z)+\frac{1}{N^{2}} G(z, z) .
$$

In this formula $\hat{\mathcal{K}}$ is a linear operator

$$
(\hat{\mathcal{K}} f)(z) \equiv \oint_{C} \frac{d \omega}{2 \pi i} \frac{V^{\prime}(\omega)}{z-\omega} f(\omega),
$$

and the contour encloses all singularities of $G(z)$, but not $z$. Note that the support $\sigma(\lambda)$ of $\rho(\lambda)$ coincides with the singularities of $G(z)$. In large $N$ limit they will be located at $s$ cuts on the real axis. If the $x_{i}, i=1, \ldots, 2 s$ denote the positions of the endpoints of 
the cuts $\left(x_{1}>x_{2}>\cdots\right)$ the solution to (16) in the large $N$ limit can be written in closed form since we can discard the term involving $G(z, z)$. We get

$$
G_{0}(z)=\frac{1}{2} \oint_{C} \frac{d \omega}{2 \pi i} \frac{V^{\prime}(\omega)}{z-\omega} \sqrt{\prod_{i=1}^{2 s} \frac{z-x_{i}}{\omega-x_{i}}}
$$

In addition the endpoints of the cuts are uniquely determined by the fact that $G_{0}(z) \sim 1 / z$ for large $|z|$, and a certain stability requirement [6]. The first requirement leads to $s+1$ equations:

$$
\frac{1}{2} \oint_{C} \frac{d \omega}{2 \pi i} \frac{V^{\prime}(\omega) \omega^{k}}{\sqrt{\prod_{i=1}^{2 s}\left(\omega-x_{i}\right)}}=\delta_{k, s}, \quad k=0, \ldots, s
$$

If we define the polynomial $M(z)$ by

$$
M(z)=\oint_{C_{\infty}} \frac{d \omega}{2 \pi i} \frac{V^{\prime}(\omega)}{(\omega-z) \sqrt{\prod_{i=1}^{2 s}\left(\omega-x_{i}\right)}}
$$

where $C_{\infty}$ is a contour at infinity in the complex plane, the requirement of stability implies [7]

$$
\int_{x_{2 k+1}}^{x_{2 k}} d \lambda M(\lambda) \sqrt{\prod_{i=1}^{2 s}\left(\lambda-x_{i}\right)}=0, \quad k=1, \ldots, s-1 .
$$

It is this kind of boundary condition which leads to the appearance of hyperelliptic integrals in formulas like (15).

Note that it follows from (18) that the planar limit of the spectral density is

$$
\rho(\lambda)=\frac{1}{2 \pi}|M(\lambda)| \sqrt{-\prod_{i=1}^{2 s}\left(\lambda-x_{i}\right)}, \quad \lambda \in \sigma(\lambda) .
$$

Having obtained the complete solution $G_{0}(z)$ in the large $N$ limit one can solve the loop equation (16) iteratively as an expansion in $1 / N^{2}$, and at a given order $h$ it is possible to construct the multi-point resolvent $G_{h}\left(z_{1}, \ldots, z_{n}\right)$ from $G_{h}(z)$. The key ingredient in this construction is the so called loop insertion operator

$$
\frac{d}{d V(z)}=-\sum_{k} \frac{k}{z^{k+1}} \frac{d}{d g_{k}}
$$

By definition it follows that

$$
G\left(z_{1}, \ldots, z_{n}\right)=\frac{d}{d V\left(z_{n}\right)} \cdots \frac{d}{d V\left(z_{1}\right)} F, \quad N^{2} F=\log Z, \quad n \geq 2 .
$$

In particular, we have

$$
G\left(z_{1}, z_{2}\right)=\frac{d}{d V\left(z_{2}\right)} G\left(z_{1}\right)
$$

\footnotetext{
${ }^{2}$ For $G(z) 1 / z$ has to be added on the r.h.s.
} 
which allows us to construct $G_{0}\left(z_{1}, z_{2}\right)$ from (18). The steps are in principle elementary, but a considerable amount of algebra is needed in order to prove that $G_{0}\left(z_{1}, z_{2}\right)$ is universal, and of the form (15) for the example of two cuts with an even potential $V(z)$. The details will be published elsewhere [8]. Let us only mention here that the main complication compared to the single cut case (i.e. $s=1$ ) is that the kernel of $\hat{\mathcal{K}}-2 G_{0}(z)$ is not zero but given by

$$
\operatorname{Ker}\left(\hat{\mathcal{K}}-2 G_{0}(z)\right)=\operatorname{Span}\left\{\frac{z^{l}}{\sqrt{\prod_{i=1}^{2 s}\left(z-x_{i}\right)}}, l=0, \ldots, s-2\right\},
$$

after taking into account the asymptotic of $G(z)$.

The $s$-cut solution will be characterized by $2 s$ classes of so called moments, given by

$$
M_{i}^{(l)}=\left.\frac{1}{(l-1) !} \frac{d^{l-1}}{d \lambda^{l-1}} M(\lambda)\right|_{\lambda=x_{i}}, \quad i=1, \ldots, 2 s .
$$

Following the derivation for $s=1$, but with considerable algebraic complications, one then can show by iteration that $G_{h}\left(z_{1}, \ldots, z_{n}\right)$ can be written in the form

$$
G_{h}\left(z_{1}, \ldots, z_{n}\right)=\frac{R_{h}\left(\left\{z_{j}-x_{i}\right\},\left\{M_{i}^{(l)}\right\},\left\{x_{i}\right\}\right)}{\sqrt{\prod_{i=1}^{2 s} \prod_{j=1}^{n}\left(z_{j}-x_{i}\right)}},
$$

where $R_{h}$ is a rational function of the poles and the moments with $1 \leq l \leq 3 h-2+n$. However, contrary to the case $s=1$ the coefficients in $R_{h}$ are no longer rational functions of the $x_{i}$, but involve hyperelliptic integrals as well.

\section{Comments}

The universal features of the single cut solution of the random matrix models generalize to an arbitrary number of cuts. As was shown in [3, 9, 10] the universality of the one-cut solution is deeply connected to the theory of intersection indices on the moduli space of punctured Riemann surfaces. It is unknown if a similar mathematical underlying structure can be related to the multi-cut solution. The fact the the coefficients in (28) can be expressed in terms of hyperelliptic integrals gives some hope in this direction, since similar

hyperelliptic integrals are encountered for the random matrix model coupled to $O(n)$ spins and this model is known to be related to integrable hierarchies.

\section{References}

[1] J. Ambjørn, J. Jurkiewicz and Y. Makeenko, Phys.Lett B251 (1990) 517.

[2] E. Brezin and A. Zee, Nucl.Phys. B402 (1993) 613.

[3] J. Ambjørn, L. Chekhov, C.F. Kristjansen and Yu. Makeenko, Nucl.Phys. B404 (1993) 127.

[4] J.C. Plefka, Nucl.Phys. B444 (1995) 333; B448 (1995) 355.

[5] J. Ambjørn, C.F. Kristjansen and Yu. Makeenko, Mod.Phys.Lett. A7 (1992) 3187. 
[6] F. David, Nucl.Phys. B348, (1991) 507.

[7] J. Jurkiewicz, Phys.Lett. B245 (1990) 178.

[8] G. Akemann, Higher genus correlators for the hermitian matrix model with multiple cuts, NBI-HE-96-30, IPT-UH-08/96, hep-th/9606004.

[9] L. Chekhov, Geometry and Physics, 12 (1993) 153.

[10] L. Chekhov, Matrix models and geometry of moduli spaces, Steklov Mathematical Institute Preprint, hep-th/9509001. 